\title{
Tap Density
}

National Cancer Institute

\section{Source}

National Cancer Institute. Tap Density. NCI Thesaurus. Code C134266.

The density, compared to the bulk density, of dry particles, expressed in mass/volume, obtained by mechanically tapping a cylinder containing the powder sample until no more change in volume occurs. 